
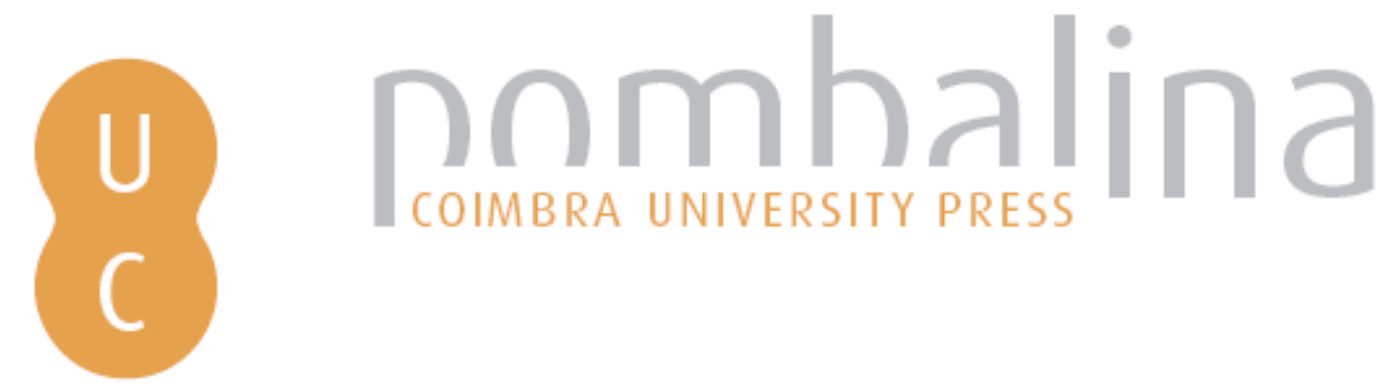

\title{
Selbstreferenz statt Subjektivität?: Probleme und folgen von Luhmanns Wissenschaftskonstruktion
}

\author{
Autor(es): $\quad$ Städtler, Michael \\ Publicado por: Imprensa da Universidade de Coimbra \\ URL \\ persistente: URI:http://hdl.handle.net/10316.2/31655 \\ DOI: $\quad$ DOI:http://dx.doi.org/10.14195/978-989-26-0205-9_20 \\ Accessed : $\quad$ 26-Apr-2023 10:22:27
}

A navegação consulta e descarregamento dos títulos inseridos nas Bibliotecas Digitais UC Digitalis, UC Pombalina e UC Impactum, pressupõem a aceitação plena e sem reservas dos Termos e Condições de Uso destas Bibliotecas Digitais, disponíveis em https://digitalis.uc.pt/pt-pt/termos.

Conforme exposto nos referidos Termos e Condições de Uso, o descarregamento de títulos de acesso restrito requer uma licença válida de autorização devendo o utilizador aceder ao(s) documento(s) a partir de um endereço de IP da instituição detentora da supramencionada licença.

Ao utilizador é apenas permitido o descarregamento para uso pessoal, pelo que o emprego do(s) título(s) descarregado(s) para outro fim, designadamente comercial, carece de autorização do respetivo autor ou editor da obra.

Na medida em que todas as obras da UC Digitalis se encontram protegidas pelo Código do Direito de Autor e Direitos Conexos e demais legislação aplicável, toda a cópia, parcial ou total, deste documento, nos casos em que é legalmente admitida, deverá conter ou fazer-se acompanhar por este aviso.

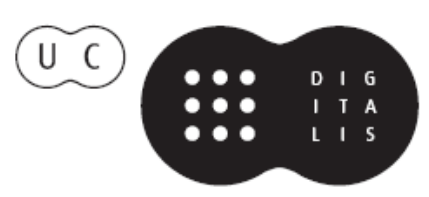


Edmundo Balsemão Pires

Burkhard Nonnenmacher

Stefan Büttner-von Stülpnagel

Editors

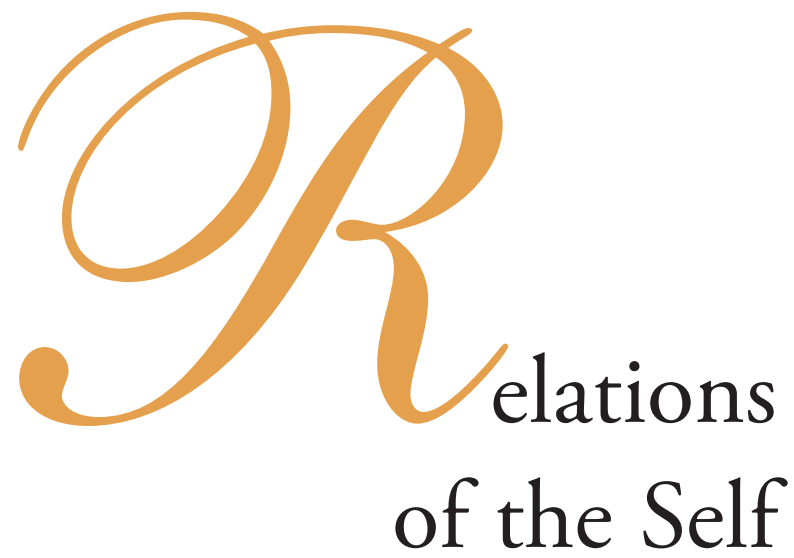




\section{Michael Städtler \\ Münster/Hannover}

\section{SELBSTREFERENZ STATT SUBJEKTIVITÄT? PROBLEME UND FOLGEN VON LUHMANNS WISSENSCHAFTSKONSTRUKTION}

„Though this be madness, yet there is method in't.“

(Shakespeare, Hamlet II,2)

\section{1) Reflexivität und Widerspruch}

Der Ausdruck ,Selbstreferenz ist im 20. Jahrhundert unter anderem an die Stelle von Begriffen wie ,Reflexion` oder ,Selbstbewusstsein“ gesetzt worden. Der Begriff Selbstreferenz ist dabei weiter, er umfasst neben dem Selbstbewusstsein auch Relationen, die nicht mit explizitem Bewusstsein von sich selbst begleitet sein müssen, wie Selbstgefühl oder Selbsterhaltung und andere mehr. Durch diese Zuordnung erfährt der philosophische Reflexionsbegriff eine Wandlung. Während nun einerseits der Versuch der Objektivierung von Subjektivität in intentionalen sprachlichen Ausdrücken zu unmittelbar reflexiven Formen zurückführte, ${ }^{1}$ beansprucht andererseits Niklas Luhmann, durch die Übertragung reflexiver

\footnotetext{
${ }^{1}$ Grundsätzlich unterstellt der Ausdruck ,Selbstreferenz' die Möglichkeit, reflexive und irreflexive Relationen gemeinsam abzuhandeln, denn ,Referenz' bezeichnet die irreflexive Relation eines Referenten auf ein Referenzobjekt, ,Selbstreferenz' dagegen bezeichnet die reflexive Relation, in der der Referent selbst zum Objekt seiner Referenz wird. Da auch diese Relation damit als Referenz gefasst wird, deren Reflexivität durch das Präfix ,selbst‘ gleichsam addiert wird, erscheint schließlich Selbstreferenz als ein logischer Sonderfall von ,Referenz überhaupt‘. - Das außerdem anzutreffende Wortmonstrum ,Selbstreflexion‘ hat, über den Gehalt des Begriffs ,Reflexion` hinaus, keinen Sinn. ,Reflexion' selbst drückt gerade die dem vernünftigen Denken wesentliche Äquivokation aus, zugleich auf etwas und auf sich selbst sich zu beziehen. - Vgl. als Initiator der neueren Diskussion in der analytischen Philosophie Sydney Shoemaker, „Selbstbezug und Selbstbewußtsein“ (d.i. „Self-Reference and Self-Awarenes"), in: Manfred Frank (Hrsg.), Analytische Theorien des Selbstbewußtseins, Frankfurt am Main 1994. Shoemaker setzt die Möglichkeit der reflexiven Prädikation in einer, self-awareness' des Prädizierenden an. Ein neuerer Versuch, das Selbstbewusstseinsproblem der analytischen Philosophie - und gleichzeitig das der von Dieter Henrich begründeten Tradition - zu lösen, ist Gunnar Hindrichs, Negatives Selbstbewußtsein. Überlegungen zu einer Theorie der Subjektivität in Auseinandersetzung mit Kants Lehre vom transzendentalen Ich, Hürtgenwald 2002. Die Arbeit beginnt problematisierend mit der Behauptung, Selbstbewusstsein sei ein Sonderfall von (intentionalem, propositionalem) Bewusstsein; das Resultat wird dann zwar durch negative Operationen gewonnen, aber nicht konsequent als
} 
Formen des Bewusstseins auf dessen Gegenstandsbereiche, also durch Subjektivierung der Objekte, sowohl Subjektivität wie Objektivität aufzulösen.

Der klassische logische Ort des Selbstbewusstseins ist die erkenntnistheoretische Reflexion, in der das formale Verhältnis der konstitutiven Momente wissenschaftlicher Erkenntnis thematisch ist. Allein diese Reflexion auf die Möglichkeit wirklicher Erkenntnisse führt das erkennende Subjekt in die antinomische Situation, zugleich Objekt zu sein, sowohl Grund als auch Begründetes zu sein, die eigene Identität nur als in sich unterschiedene denken zu können. ${ }^{2}$ Dabei ist es diese Identität des Selbstbewusstseins, die als Bedingung der Identität der Erkenntnisse erschlossen wird. Das Selbstbewusstsein muss seine Einheit gegen den eigenen inneren Widerspruch als Forderung der Widerspruchsvermeidung festhalten, wenn es überhaupt etwas erkennen will. Ohne diesen grundsätzlichen Widerspruch im Selbstbewusstsein wäre wohl der Satz vom zu vermeidenden Widerspruch gar nicht formulierbar. Wohlgemerkt: All dies - Widerspruch im Selbstbewusstsein und Forderung der Identität durch Widerspruchsvermeidung - kann erkenntnistheoretisch nicht als Ursprung von Wissenschaft in Anspruch genommen werden, aber es kann als deren Bedingungen erschlossen werden, wenn es Wissenschaft in Form systematisch verbundener sachhaltiger universaler Urteile schon gibt.

\section{2) Probleme der Wissenschaftsvorstellung Niklas Luhmanns: Auflösung der Objektivität}

Für Niklas Luhmann gehört es nun „zu den schlimmsten Eigenschaften unserer Sprache [...], die Prädikation auf Satzsubjekte zu erzwingen und so die Vorstellung zu suggerieren [...], daß es um ,Dinge' gehe, denen irgendwelche Eigenschaften [...] zugeschrieben werden “3. Statt dessen sei in der Systemtheorie eben „nicht ein Gegenstand [...], sondern die Differenz von System und Umwelt" ${ }^{\star 4}$ thematisch. Diese Differenz will Luhmann als Selbstkonstitution

Reflexionsbegriff behandelt. Der Grundfehler der gesamten Debatte ist die Grammatikalisierung von Selbstbewusstsein. Grammatische Reflexivität hat immer schon die Form von Urteilen, deren Formulierung Selbstbewusstsein schon voraussetzt. Shoemaker, wie viele andere, bemerkt diesen Vorrang, kann ihn aber auf der Ebene grammatischer Analyse nicht begründen. Dies hat Dieter Henrich einmal als „versachlichte Analyse der Sprache“ kritisiert: „Fichtes ursprüngliche Einsicht“, in: Subjektivität und Metaphysik, hrsg. von Dieter Henrich und Hans Wagner, Frankfurt am Main 1966, S. 188. - Dass durch die Objektivierung des Selbstbewusstseins in dessen Reflexionsmodell das Selbstbewusstsein zu einem Sonderfall von Bewusstsein werde, vertritt auch Karen Gloy, „Der Begriff des Selbstbewußtseins bei Kant und Fichte“, in: Dietmar H. Heidemann (Hrsg.), Probleme der Subjektivität in Geschichte und Gegenwart, Stuttgart 2002, S. 127: Kant „importiert [...] die intentio recta in das Selbstbewußtsein, biegt sie zur intentio obliqua um und deklariert so das Selbstbewußtsein zu einem Sonderfall von Gegenstandsbewußtsein“. Da der Begriff des Selbstbewusstseins bei Kant ein ganz negativer, reflektierter ist, der eben kein Objekt bezeichnet, sondern bloß eine logische Funktion zu denken, trifft ihn dieser Vorwurf sicher nicht; er trifft wahrscheinlich nicht einmal Kants Konzept von Selbsterkenntnis, die sich ihr Objekt selbst in der reinen Anschauung geben soll.

${ }^{2}$ Vgl. Dieter Henrich, „Fichtes ursprüngliche Einsicht“, a.a.O., S. 192. Henrich zeigt, dass Fichte für diesen Grundwiderspruch viele Formulierungen hat (vgl. S. 224, S. 213ff.).

${ }^{3}$ Niklas Luhmann, Soziale Systeme. Grundriß einer allgemeinen Theorie, Frankfurt am Main 1987, S. 115 und S. 595. Dieses Zitat weist Peter V. Zima zufolge Luhmann als postmodernen Denker aus. Vgl. Theorie des Subjekts. Subjektivität und Identität zwischen Moderne und Postmoderne, Tübingen 2000, S. 324, S. 326.

${ }^{4}$ Niklas Luhmann, Soziale Systeme, a.a.O., S. 116. 
des Gegenstandes verstehen, der sich durch Unterscheidung von anderem erst als er selbst konstituiere. Damit aber ist das, was der Gegenstand selbst wäre, aufgelöst in Relationalität ohne Relata: Die Relata sollen ja erst durch die Relation gesetzt werden.

Die - wie Luhmann es nennt - ,Supertheorie' als Theorie davon, was Theorien seien, könne nun ganz unabhängig von bestimmten Theorien aufgebaut werden, weil schon die ,Normaltheorien“ - Luhmanns Ausdruck für Einzelwissenschaften - ihre Gegenstände nicht prinzipiell erklärten, sondern als reine Relationalität reformulierten, die als solche gehaltlos und deswegen scheinbar beliebig auszufüllen seien: In Unterscheidung zu welcher Negation seiner selbst der Gegenstand sich konstituiere, könne durch kein begründetes Kriterium vorab festgelegt werden. So könnten „nicht nur gegenstandsbezogene Theorien, sondern auch gegenstandskonstituierende Kriterien einander ablösen“" und Wissenschaft „verzichtet [...] auf a priori geltende Prüfbedingungen für das, was Erkenntnis ist, und gibt [...] [diese] der historischen Relativierung preis“6. Die Systemtheorie ist somit eine Methode nicht zur Erkenntnis, sondern zur Konstruktion von Gegenständen. Da diese Methode ihren Gegenständen vorhergeht, ist sie selbst gegenstandslos, d.h. unbestimmt und kann daher zu gegensätzlichen Resultaten führen. Die ,Supertheorien' betrachteten daher nicht, welche Theorie ihren Gegenstand richtig erkläre, sondern sie setzten ,konkurrierende 'Theorien deren gesellschaftlicher Funktion nach ins Verhältnis und fassten sie als funktionale Teilsysteme des sich selbst ausdifferenzierenden Wissenschaftssystems. Luhmann selbst zufolge kopiert dieses Verfahren formal das der Ideologiekritik, jedoch ohne Wahrheitsanspruch.?

"Supertheorien relationieren also Relationen zum Gegenstand. "8 Eine ,Supertheorie“ ist damit ein Verfahren, Relationen von Relationen zu bilden, „für das sie selbst die Kriterien beschafft" . Die jeweilige Auswahl der Kriterien diene dazu, „die Position des Wissenschaftssystems in bezug auf andere Umweltausschnitte (etwa Wirtschaft, Politik, Motivation des wissenschaftlichen Personals) [zu] erhalten bzw. [zu] verbessern "10. Was zum Gegenstand gemacht wird und wie es zum Gegenstand gemacht wird, folgt dann nicht dem Zweck der Gegenstandserkenntnis, sondern wissenschaftsfremden Zwecken, zunächst der ökonomischen oder politischen Opportunität. Ins Wissenschaftssystem fällt dies allein deshalb noch, weil Luhmann unter diesem Titel Wissenschaft mit Wissenschaftsbetrieb verwechselt. ${ }^{11}$ Das deutet das dritte Kriterium, die ,Motivation des wissenschaftlichen Personals', an: Ist solche Motivation nötig, so hat dieses ,Personal' entweder kein Interesse an wissenschaftlicher Arbeit, hat also den falschen Beruf ergriffen, oder es hat zwar wissenschaftliches Interesse, muss aber aus fachfremden Gründen dazu motiviert werden, dieses vorhandene wissenschaftliche Interesse fachfremden Zwecken unterzuordnen. Für die zweite Erklärung spricht Luhmanns

\footnotetext{
${ }^{5}$ Niklas Luhmann, „Soziologie der Moral“, in: Ders., Die Moral der Gesellschaft, Frankfurt am Main 2008, S. 59

${ }^{6}$ Niklas Luhmann, „Soziologie der Moral“, a.a.O., S. 60.

${ }^{7}$ Niklas Luhmann, „Soziologie der Moral“, a.a.O., S. 68.

${ }^{8}$ Niklas Luhmann, „Soziologie der Moral“, a.a.O., S. 74.

${ }^{9}$ Niklas Luhmann, „Soziologie der Moral“, a.a.O., S. 74.

${ }^{10}$ Niklas Luhmann, „Soziologie der Moral“, a.a.O., S. 75.

${ }^{11}$ Dass diese Verwechslung intendiert ist, führt Luhmann aus in: „Die Praxis der Theorie“, in: Ders., Soziologische Aufklärung. Aufsätze zur Theorie sozialer Systeme, Opladen, 1970. Vgl. auch dens., Soziale Systeme, a.a.O., S. 655ff.
} 
wissenschaftstheoretisches Vokabular, das sich von „Strategien“ über „Taktik“ und „Konkurrenzlagen“ oder „Theorie-Angebote“ mit „Gewinnmarge“ bis zum „verfeinerten Arrangierbewußtsein" erstreckt.

Die aus der Verknüpfung von Wissenschaft, kontingenter Konstitution von Gegenständen und wissenschaftsfremden Konstitutionskriterien folgende ,Konkurrenz' entgegengesetzter Theorien bedürfe nun um der Stabilität des Wissenschaftssystems willen der Kontrolle. Diese sollen eben ,Supertheorien' leisten, die als selbstreferentielle Systeme auf Theorien referieren. Sie selbst seien dann bloß ein ,Fall von Theorie', eben die Theorie von Theorien. ${ }^{12}$ Der eminente Unterschied von Theorie und Gegenstand ist damit eingeebnet. Erkenntnistheorie kann dagegen seit Platons Charmides wissen, dass sie keine wissenschaftliche Erkenntnis ist, dass ihr Gegenstand nicht mit den Gegenständen der Wissenschaften gleichrangig ist und dass sie folglich selbst nicht zu ihren Gegenständen gehört, sondern ihren Ort in der notwendig widerspruchsvollen Reflexion des erkennenden Subjekts hat. Den Widerspruch der erkenntnistheoretischen Reflexion überträgt Luhmann nun aber unter dem Titel der Selbstreferenz auf die Gegenstände der Wissenschaft: „Dabei wird der Begriff der Selbstreferenz (Reflexion, Reflexivität) von seinem klassischen Standort im menschlichen Bewußtsein oder im Subjekt gelöst und auf Gegenstandsbereiche, nämlich auf reale Systeme als Gegenstand der Wissenschaft übertragen." ${ }^{13}$ Das Denken soll Gegenstände so konstituieren, als ob sie sich selbst erzeugten: „Damit gewinnt man zugleich eine gewisse Distanz zu den rein logischen Schwierigkeiten der Selbstreferenz. Diese bedeuten dann nur noch: daß es in der wirklichen Welt Systeme gibt, deren Beschreibung durch andere Systeme in diesen (!) zu unentscheidbaren logischen Widersprüchen führt. "14 Indem die Form des erkenntnis-

${ }^{12}$ Niklas Luhmann, „Soziologie der Moral“, a.a.O., S. 60.

${ }^{13}$ Niklas Luhmann, Soziale Systeme, a.a.O., S. 58. Der Widerspruch findet sich grundsätzlich in der Auffassung, offene Systeme könnten nur aufgrund ihrer Geschlossenheit offen sein. Nur weil sie sich auf sich selbst bezögen, könnten sie sich auf anderes beziehen. Vgl. Soziale Systeme, a.a.O., S. 606 und S. 624. - Luhmann behauptet nun freilich, der Subjektbegriff sei seinerseits Resultat einer Übertragung der objektiven Selbstreferenz auf ein transzendental bestimmtes Bewusstsein. (Vgl. Niklas Luhmann, Soziale Systeme, a.a.O., S. 606f. und dens., „Soziologie der Moral“, a.a.O., S. 92f. Diese ,Überanstrengung' des Subjektbegriffs sei geschichtlich der Anlass für die Systemtheorie. Vgl. a.a.O., S. 87f.) Die Gegenbehauptung wäre nun eine trockene Versicherung gegen eine andere, wenn nicht der Begriff von Reflexion nur als Resultat erkenntnistheoretischer Reflexion möglich wäre und deswegen - worauf immer er Anwendung finden mag - außerhalb des Zusammenhangs erkenntnistheoretischer Reflexion sinnlos würde. Luhmanns Behauptung, dass es selbstreferentielle Systeme in der Realität gebe, beschwört die Ontologie, der sie abzuschwören vorgibt. Vgl. „Soziologie der Moral“, a.a.O., S. 93, S. 160. Die Welt erscheint schließlich, in kruder Travestie Kants, als Organismus, aus dem durch Projektion kausaler und logischer Formen nach Bedarf Gegenstände auszuschneiden wären. Vgl. Soziale Systeme, a.a.O., S. 648ff.

${ }^{14}$ Niklas Luhmann, Soziale Systeme, a.a.O., S. 58. Ob es ,in der wirklichen Welt' Systeme, selbstreferentielle Prozesse, gibt, darf bezweifelt werden. Was selbst auf sich selbst referiert, muss zunächst wohl mit Bewusstsein es selbst sein. Sonst sind es Naturprozesse, die so beschrieben werden können, als ob sie sich auf sich bezögen. Kant hat mit großen Problemen dafür argumentiert, dass man sie so beschreiben müsse; auch diese Probleme überspringt Luhmanns Behauptung im ontologischen Duktus noch. - Ein System ist erkenntnistheoretisch ein notwendiger Zusammenhang, der als Ganzer und in seinen Elementen aus einem begrifflichen Prinzip bestimmt und daher geschlossen ist. Das dürfte auf keinen der originären Gegenstände Luhmanns zutreffen; die Konstruktionen der operativen Geschlossenheit bei funktionaler Offenheit, der Anschlussfähigkeit, der Kopplungen usw. sprechen dafür. Luhmann verwendet einen spezifisch naturwissenschaftlichen, vor allem biologischen, Systembegriff, den er auf soziale Zusammenhänge anwendet. Er teilt damit eine Eigenschaft der langen Tradition des organischen Politikmodells: Es war immer schon falsch, wo Zwecke, nicht Funktionen wirken. 
theoretischen Selbstbewusstseins auf Erkenntnisgegenstände übertragen wird, werden diese nicht etwa erkennbar, sondern expressis verbis unerkennbar. Sie erscheinen im Denken als Widersprüche. Umgekehrt soll diese vom Denken selbsterzeugte und in die Gegenstände projizierte Widersprüchlichkeit für den erkenntnistheoretischen Widerspruch im Selbstbewusstsein einstehen. Auf dieser Grundlage kann es der Theorie nicht mehr um Lösung des Widerspruchs gehen, sondern um „Zusatzvorkehrungen für Anschlußfähigkeit"15. In der Tat vertritt Luhmann, dass die Konstrukteure von ,Supertheorien“ sich beliebig für einen Systemgrundsatz entschieden, den sie dann durch Anschlussbestimmungen erst inhaltlich auffüllten. ${ }^{16}$ Damit kehrt Luhmann den Prozess erkenntnistheoretischer Reflexion um, ohne ihn zuvor beschritten zu haben. Der Grundsatz müsse dafür nur eine Bedingung erfüllen, er muss die Form reiner Differenz haben. Da nun der absolute Unterschied direkt auf den absoluten Widerspruch führt, lässt sich an einen solchen Systemgrundsatz alles Beliebige anschließen; vor allem aber gewährt der Widerspruch im Grundsatz, dass dieser das angeschlossene ,System' nicht durch Wahrheitsanspruch dominiert, sondern selbst durch dessen Inhalte bestimmbar bleibt, - also gar kein Grundsatz ist. Da Luhmann inkonsequent diesen gegenstandslosen Theoriebegriff auf bestimmte Gegenstände bezieht, dienen die weiteren Systemfunktionen dazu, auch miteinander inkohärente Dinge formal kohärent darstellbar zu machen, indem sie aufgrund, zeitlicher Kontingenz' beständig umarrangiert werden: Da sie so nie in ein logisch bestimmtes Verhältnis geraten, entsteht der Anschein, sie seien miteinander verträglich.

Damit ist ein Wissenschaftsbetrieb beschrieben, in dem alle Meinungen sich zueinander ins Verhältnis setzen können, weil sie nur erst aus diesem Verhältnis heraus sich selbst als Meinungen positionieren und dabei noch wechselnden Moden folgen. - Wenn nun Naturwissenschaft und Technologie all das, was Luhmann preisgibt, preisgäben, käme die materielle Reproduktion der Gesellschaft zum erliegen. ${ }^{17}$ - Dass in den Geistes- und Gesellschaftswissenschaften dagegen solche Preisgabe überhaupt möglich ist, hat einen Grund, den Luhmann gesehen hat: „Auf den Normalebenen theoriegeleiteter Forschungsarbeit wird man erkenntnistheoretische Voraussetzungen und moralische Ansprüche nicht eigens reflektieren. Die operativen Entscheidungen, die man zu treffen hat, erfordern dies nicht. "18 Die Wissenschaften bedürfen der Erkenntnistheorie nicht, weil ihr Erkenntnisverfahren durch Formen des Denkens und der Gegenstände bestimmt wird. ${ }^{19}$ Umgekehrt setzen aber

${ }^{15}$ Niklas Luhmann, Soziale Systeme, a.a.O., S. 59.

${ }^{16}$ Vgl. Niklas Luhmann, Soziale Systeme, a.a.O., S. 78. Diese Systembauweise, in der um einen vorausgesetzten inneren Grundsatz alle hinzutretenden äußeren Bestimmungen durch rein spekulative Anstrengung passend arrangiert werden, unterscheidet sich formal nicht von der Konstruktion paranoid-schizoider Wahnsysteme. Nur ist das pathologische Bewusstsein, selbst wenn es noch auf den Systembildungsprozess reflektiert, nicht mehr in der Lage, den Systemgrundsatz beliebig zu wechseln.

${ }^{17}$ Der leichtfüßige Umgang mit Kausalität und Logik, den Luhmann für wissenschaftliche Realität hält, wird weder in Naturwissenschaft noch in Technologie praktiziert. Vgl. Niklas Luhmann, Soziale Systeme, a.a.O., S. 634.

${ }^{18}$ Niklas Luhmann, „Soziologie der Moral“, a.a.O., S. 57.

${ }^{19}$ Übrigens bedarf auch die gesellschaftliche Wirklichkeit von Wissenschaft zur Vermittlung ihrer Positionen nicht der soziologischen ,Supertheorie‘. Die realen Widersprüche werden durch gesellschaftliche oder politische Gewalt vermittelt. - Gewalt wird bei Luhmann kaum erwähnt. Vgl. Peter V. Zima, Theorie des Subjekts, a.a.O., S. 326 und S. 345. 
Geistes- und Gesellschaftswissenschaften systematisch und existentiell voraus, dass eindeutig bestimmte wissenschaftliche Naturerkenntnis möglich und wirklich ist. Sonst wären Menschen nicht in der Lage, sich als selbstbewusste Subjekte vom Kontinuum der Naturkausalität zu unterscheiden. Der Versuch, diese Differenz von Mensch und Natur unter dem Titel selbstreferentieller Systeme wieder einzuziehen, kann die eigenen Voraussetzungen ignorieren, weil das Voraussetzungsverhältnis von Naturerkenntnis und Selbsterkenntnis nicht reziprok ist. Die Trennung von Natur- und Geisteswissenschaften schließt das Vergessen der materiellen Bedingungen von geisteswissenschaftlicher Erkenntnis ein, löst diese von der Vernunft und öffnet sie der freien Willkür.

\section{3) Der erkenntnistheoretische Hintergrund: Modelle}

Obwohl demnach wissenschaftliche Erkenntnis der erkenntnistheoretischen Reflexion nicht bedarf, ist diese doch nicht gegenstandslos, jedenfalls dann nicht, wenn sie sich als Reflexion schon bestimmter Erkenntnis weiß. Indem die Subjekte aus dem Verhältnis von Einheit und Unterschied im Selbstbewusstsein den Satz vom zu vermeidenden Widerspruch gewinnen können, vermögen sie in grundsätzlicher Form Wissen von Meinungen zu unterscheiden. Der Begriff der Reflexivität des Subjekts wird so zur Grundlage kritischen Selbstbewusstseins. Die philosophische Bemühung allerdings, diese Grundlage kritischen Selbstbewusstseins ihrer logischen Form nach zu begreifen, führt prinzipiell die Tendenz zur Verselbständigung erkenntnistheoretischer Reflexion in absolute subjektlose Reflexivität mit sich.

\section{a) Aristoteles}

Schon Aristotele ${ }^{20}$ gelangt von der Frage, wie das Einzelne trotz Vielfalt und Veränderung bestimmt zu erkennen sei, zu der reflektierten Lösung, alle singulären Veränderungen im allgemeinen Begriff von Veränderlichkeit aufzuheben. Dieser sei zudem in sich so differenziert, dass die selbstbezügliche Veränderlichkeit ihre Selbstbeziehung gerade dadurch herstelle, dass sie von ihr Unterschiedenes derart in Veränderung versetze, dass dieses wieder seinen Impuls an den Ursprung zurückgebe. Damit ist der widersprüchliche Begriff eines kinoun akineton, eines unbewegt bewegenden Prinzips, gegeben, das als subjektlose reine Reflexivität gedacht werden kann. ${ }^{21}$ Die intelligible Bestimmung dieser Reflexion als noäsis noüseos -

\footnotetext{
${ }^{20}$ Für eine detaillierte Untersuchung zu den hier nur angerissenen Reflexionstheorien bei Aristoteles, Thomas von Aquin und Hegel vgl. Michael Städtler, Die Freiheit der Reflexion. Zum Zusammenhang der praktischen mit der theoretischen Philosophie bei Hegel, Thomas von Aquin und Aristoteles, Berlin 2003.

${ }^{21}$ Vgl. Aristoteles, Metaphysik, Hamburg 1991, 1072 a: „Also war nicht eine unendliche Zeit Chaos oder Nacht, sondern immer dasselbige [...]. Wenn nun immer dasselbe im Kreislauf besteht, so muß etwas bleiben, das gleichmäßig in wirklicher Tätigkeit ist. Soll aber Entstehen und Vergehen vorhanden sein, so muß etwas anderes existieren, was in anderer und wieder anderer Weise wirklich tätig ist. Es muß also in der einen Weise in Beziehung auf sich selbst, in der
} 
Denken des Denkens - weist sie sowohl als erkenntnistheoretische Reflexion als auch als reine Reflexivität aus. ${ }^{22}$

\section{b) Thomas von Aquin}

Der Aristoteliker und Neuplatoniker Thomas von Aquin rekonstruiert die erkenntnistheoretische Reflexion im Zusammenhang der Trinitätslehre. ${ }^{23}$ Sich selbst könne der Intellekt wohl erkennen, indem er sich selbst zum Objekt werde, aber dafür müsse er sich in sich unterscheiden. Dafür sei aber eine inhaltliche Bestimmung vorausgesetzt, die der Intellekt nicht aus sich selbst, sondern aus der Erkenntnis von ihm unterschiedener Gegenstände habe. Selbsterkenntnis sei daher nicht als absolute Reflexivität, sondern erst als relational vermittelte möglich. Aber diese relational aufgelöste Reflexion genüge nicht, denn jeder Mensch habe doch eine unmittelbare Kenntnis seiner selbst und höre nicht auf, er selbst zu sein, wenn er etwa schlafe. Diesen Grund im Bewusstsein, diese ursprüngliche Einsicht, nennt Thomas ,habituelle Selbsterkenntnis', durch die jeder Intellekt wisse, dass er sei, auch ohne zu wissen, was er sei. Diese Unmittelbarkeit entzieht sich der Darstellung. Sie wird aber darstellbar an dem vom menschlichen Subjekt abgelösten Modell der trinitarischen Reflexivität Gottes. ${ }^{24}$

Gott sei als Gott-Vater reine Selbstbeziehung, reine Wirklichkeit des Wissens seiner selbst. Diese absolute Reflexivität erfülle er durch Aussprechen oder Vorstellen seiner selbst in dem Sohn, der das Wort, also die bestimmte Vorstellung, Gottes selbst sei. Im Sohn schaue Gott sich selbst als von ihm unterschieden und dadurch bestimmt an. Die setzende Reflexion werde sich selbst durch sich selbst zur äußeren: „Indem nämlich Gott-Vater sich selbst denkt, empfängt er das Wort. ${ }^{\text {"25 }}$ Die Reflexivität im Sohn sei nun die gleiche wie die

anderen Weise in Beziehung auf anderes wirken, und dies also in Beziehung auf ein verschiedenes drittes oder auf das erste. Notwendig auf dies; denn dies ist wieder sich selbst wie jenem anderen Ursache der Bewegung.“

${ }^{22}$ Einerseits wird die noäsis noäseos als ,göttlich', d.h. nicht als personaler Gott, aber doch absolut bezeichnet, andererseits verweist dieser Ausdruck auf die Darstellung menschlicher Reflexivität in Aristoteles, Über die Seele, übers. von Horst Seidel, Hamburg 1995, Buch III, Kap. 5.

23 Thomas entwickelt in Quaestiones disputatae de Veritate, übers. von Edith Stein, Louvain 1952, Bd. 1, quaestio X (De Mente) die Bestimmungen des menschlichen Erkenntnisvermögens von der Erkenntnis der materiellen Dinge über die Selbsterkenntnis bis zur Gotteserkenntnis. Dabei wird einerseits der menschliche Geist als trinitarisch in Verstand, Gedächtnis und Wille gegliederter entwickelt und andererseits der allgemeine Begriff göttlicher Trinität inhaltlich entfaltet. Die abschließende Behauptung der Unerkennbarkeit Gottes in statu viae folgt einer recht genauen Analyse dessen, was nicht erkennbar sei. - In der Summa theologiae werden beide Gegenstandsbereiche zwar räumlich getrennt abgehandelt, stehen aber in engem sachlichem Verhältnis.

${ }^{24}$ Zur Entwicklung dieses Problems von der Reflexion zum Grundreflex bei Fichte vgl. Dieter Henrich, „Fichtes ursprüngliche Einsicht“, a.a.O., S. 226, Anm. 41. In Antizipation Fichtes könnte man sagen, in der Thomasischen Lösung des Unmittelbarkeitsproblems durch die Theorie göttlicher Reflexion sei diese als absolutes Sich-setzen, als Tathandlung Gottes zu denken. Fichte ist daher nicht der erste gewesen (vgl. a.a.O., S. 195), der dieses Problem erkannt hat und er ist es eben auch nicht gewesen, der gesehen hätte, dass absolutes Selbstbewusstsein zwar notwendiges Moment von Selbstbewusstsein ist, aber - paradox - nicht als absolutes darstellbar ist, ohne die Widersprüche der Reflexion positiv zu setzen.

${ }^{25}$ Thomas von Aquin, Der Prolog des Johannisevangeliums, übers. von Wolf-Ulrich Klünker, Stuttgart 1986, I. 
im Vater, und doch seien beide asymmetrisch in Beziehung aufeinander, denn die Vaterschaft sei nicht die Sohnschaft. Vater und Sohn sind in ihrer Reflexion in sich identisch, so dass kein Polytheismus entsteht. Die mit der Identität der Relata drohende logische Implosion des relationalen Gottesbegriffs soll nun vermieden werden durch eine Differenz in den Relationen des Vaters zum Sohn und des Sohns zum Vater. Da beide in sich identisch sind, kann die Differenz der Relationen nicht in den Relata gründen, sondern nur in geheimnisvoller Weise in den Relationen selbst. So liege auch die Personalität letztlich paradoxerweise nicht in den Personen, sondern in ihren Relationen aufeinander: „Die Person nämlich bezeichnet die Beziehung als etwas, das in göttlicher Natur für sich bestehend ist [relatio in se subsistens]. "26 Das Wesen Gottes wird damit als Reflexion verstanden, die sich selbst absolut-relational auslegt, - und dadurch doch wieder bei sich bleibt. Allerdings sei Gott nicht bloß Selbsterkenntnis, sondern zugleich absolutes Wollen seiner selbst. Auch dieses Wollen erwirke von Ewigkeit die Erzeugung des Sohnes, weil Gott sich selbst nur wollen könne, wenn er bestimmtes Wissen von sich habe: Was man nicht kennt, kann man nicht erstreben. Weil übrigens dieses bestimmte Wissen und Wollen seiner selbst in sich zusammenbräche, konstituieren Vater und Sohn gemeinsam die sie verbindende Liebe zum heiligen Geist, der dritten absoluten Reflexionsgestalt, durch die die göttliche Reflexion als bestimmende in die Welt - die es dann auch geben muss ausstrahlt. $^{27}$

\section{c) G.W.F. Hegel}

Die in diesem Reflexionsmodell liegende Koinzidenz von Subjektlosigkeit und Produktivität fasst Hegel schließlich rein logisch. Die Gegenstandslosigkeit der absoluten Reflexion, die Hegel aus dem Verhältnis von Wesentlichem und Unwesentlichem entwickelt, nötigt die Reflexion, die Bedingung ihrer Bestimmtheit selbst zu setzen. Da die setzende Reflexion aber aus sich selbst nichts als wieder sich selbst setzen kann, ergibt sich eine ,äußere Reflexion“ als notwendiges Moment bestimmter Reflexivität. Sie soll der reflektierenden Vermittlung die Unmittelbarkeit zuführen, aus deren Vereinigung dann Reflexion als bestimmende Bestimmungen setzende - resultiere.

Die Assoziation erkenntnistheoretischer Reflexion weist Hegel ausdrücklich zurück: Es sei „weder von der Reflexion des Bewußtseyns, noch von der bestimmtern Reflexion des Verstandes, die das Besondere und Allgemeine zu ihren Bestimmungen hat, sondern von der

${ }^{26}$ Thomas von Aquin, Summa theologiae. Die Deutsche Thomas-Ausgabe, hrsg. von Dominikanern und Franziskanern Deutschlands und Österreichs, Salzburg u.a. 1933ff., I, 39, 1 c.

${ }^{27}$ Luhmanns gelegentliche philosophiegeschichtliche Ausflüge (vgl. z.B. Niklas Luhmann, Soziale Systeme, a.a.O., S. 615) sollen die Bedeutung der Reflexion im Übergang zur Neuzeit im Rahmen seines an Durkheim anschließenden Konzeptes funktionaler Differenzierung, also als Funktion gesellschaftlicher Entwicklung erklären, die nicht durch Subjekte hervorgebracht sei. Mit etwas Arbeit am Gegenstand ließe sich zeigen, dass es in theoretischer wie in praktischer Hinsicht das subjektive Bewusstsein von objektiven Aporien ist, das die Entwicklungen bestimmt. 
Reflexion überhaupt die Rede“28. - Tatsächlich gewinnt die setzende Reflexion jene Äußerlichkeit allein aus Selbstunterscheidung, „sie bezieht sich auf sich als auf jenes ihr Nichtseyn" ${ }^{29}$. Damit ist das Subjekt der Reflexion allein diese selbst; sie bleibt als setzende Reflexion auch das Subjekt der äußeren, durch die sie einmal überprüft, was wäre, wenn sie nicht absolut wäre. Da sie aber Subjekt dieses Experiments bleibt, ist es vorab schon entschieden.

Das Subjekt der begrifflichen Entwicklung in der Wissenschaft der Logik sind die Begriffe selbst, letztlich ist es der Begriff schlechthin, der sich in Form von Reflexionsbewegungen - Selbstunterscheidung und Selbstbeziehung - durch verschiedene kategoriale Gestalten zur absoluten Idee bewegt. Dem entspricht es, dass in der Lehre vom Begriff, dem eigentlichen Ort von Subjektivität in der Logik, Subjektivität und Objektivität als Momente des Begriffs ineinander aufgelöst werden. Subjektivität wird zu dem Moment, durch das der Begriff selbst zur Objektivität übergeht: „[...] Objectivität hat der Gegenstand somit im Begriffe, und dieser ist die Einheit des Selbstbewußtseyns, in der er aufgenommen worden; seine Objectivität oder der Begriff ist daher selbst nichts anderes als die Natur des Selbstbewußtseyns, hat keine anderen Momente oder Bestimmungen als das Ich selbst. "30 Dass Hegel Objektivität auf das ,Ich' zurückführt, weist aber nicht dessen Vorrang aus, denn Subjekt dieser Entwicklung ist wieder der Begriff selbst, der mittels seines subjektiven Momentes „sich selbst zur Sache [macht] und [...] dadurch das Verhältnis der Subjectivität und Aeusserlichkeit gegen sie“31 verliere. Der Begriff, analog der Reflexion, überwindet durch sein subjektives Moment seine Subjektivität. So soll der Begriff subjektlos in sich selbst sich vermitteln, bis er „durch die in ihm selbst gegründete Dialektik zur Realität so übergeht, daß er sie aus sich

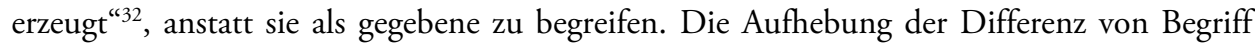
und Realität in der subjektlosen absoluten Reflexion der Idee führt auf Probleme, die sich an der von Hegel ausgeführten Idee des Staates demonstrieren lassen:

In dieser wird ein Kollektiv von Subjekten so organisiert gedacht, dass deren partikulare Interessen und Pflichten mit den Interessen und Aufgaben des Allgemeinen vollständig vermittelt seien. Der Staat wird damit zu einem System, das sich in den Beziehungen der zu seinen Momenten vermittelten Einzelnen immer auf sich selbst bezieht und durch diese Reflexion sich selbst erhält und weiter bestimmt, ohne dass in diesem Prozess noch selbständige Subjekte agierten. Bis hin zum Monarchen sind alle Gewalten und Institutionen

${ }^{28}$ G.W.F. Hegel, Wissenschaft der Logik. Lehre vom Wesen, in: Ders., Gesammelte Werke (GW), hrsg. von der Rheinisch-Westfälischen Akademie der Wissenschaften, Bd. 11, Hamburg 1978, S. 254. Hier wäre mit Karen Gloy der idealistische Selbstbewusstseinsbegriff als ,nicht-egologisch` von dem ,egologischen Kants' zu unterscheiden: Prinzip der Philosophie ist nicht ein Ich, sondern eine Selbstbewegung, die, vor allem bei Fichte, noch den Titel ,Ich“ tragen kann. Vgl. Karen Gloy, „Der Begriff des Selbstbewußtseins bei Kant und Fichte“, a.a.O., S. 133ff., bes. S. 138. - Vgl. auch Otto Pöggeler, „Die Fehlstelle in Hegels Logik der Subjektivität“, in: Dietmar H. Heidemann (Hrsg.), Probleme der Subjektivität in Geschichte und Gegenwart, a.a.O., S. 269: „Da die Ausrichtung auf Gewißheit nicht verloren gehen darf, muß nach Kant ein ,Ich denke' alle begriffliche Arbeit begleiten. Darf Hegels Wissenschaft der Logik aber den sich bewegenden Gedanken das Denken in seiner Selbstbezüglichkeit hervortreten lassen [...]?“

${ }^{29}$ G.W.F. Hegel, Wissenschaft der Logik. Lehre vom Wesen, a.a.O., S. 253.

${ }^{30}$ G.W.F. Hegel, Wissenschaft der Logik. Lehre vom Begriff, in: Ders., Gesammelte Werke (GW), hrsg. Von der Rheinisch-Westfälischen Akademie der Wissenschaften, Bd. 12, Hamburg 1981, S. $18 f$.

${ }^{31}$ G.W.F. Hegel, Wissenschaft der Logik. Lehre vom Begriff, a.a.O., S. 30.

${ }^{32}$ G.W.F. Hegel, Wissenschaft der Logik. Lehre vom Begriff, a.a.O., S. 25. 
funktionelle Momente. Als solche sind sie austauschbar und gegen die Existenz der Idee gleichgültig: „Der schlechteste Staat, dessen Realität dem Begriffe am wenigsten entspricht, insofern er noch existirt, ist er noch Idee; die Individuen gehorchen noch einem Machthabenden Begriffe. "33 Der kritische Einwand, der Staat solle aber seinem Begriff entsprechen, setzte voraus, dass dieser Begriff in relativer Unabhängigkeit zu den historischen Staaten seinen Ort in der Reflexion der Subjekte hätte und diesen als Maßstab der Kritik zur Verfügung stünde.

Die Entsubjektivierung der Reflexion folgt bei Hegel dem Zweck, den Begriff der Reflexivität als Reflexivität des Begriffs rein philosophisch konsequent zu Ende zu denken. Das noch aufklärerisch inspirierte kritische Potential des Begriffs, seine Idealität gegen die mangelhafte Realität zu richten, geht aber in dieser Konsequenz verloren, weil die philosophische Reflexion den spezifischen Boden ihrer Reflexivität, die Reflexion auf bestimmte Erkenntnis, verlässt.

\section{4) Folgen der Wissenschaftsvorstellung Niklas Luhmanns: Auflösung der Subjektivität}

Luhmann nimmt dieses widersprüchliche Resultat der Philosophiegeschichte als deren positives Ergebnis und zieht daraus die von allen bestimmten Bedingungen abstrahierte Vorstellung der Selbstreferenz, die noch den Unterschied von Subjekt und Objekt, der für Hegel trotz allem konstitutiv war, in substanz- und substratlose Relationen auflöst. Das dialektisch bestimmte Verhältnis beider wird zum abstrakt methodologischen Arrangement. ${ }^{34}$ Zudem bewegt dieses Arrangement sich ganz von selbst. Subjektivität wird nicht sowohl aufgehoben als vielmehr zu einem „abstrakten Subjekt-Aktanten“"35 mythisiert. Das mag einen Grund darin haben, dass die ideologische Form gesellschaftlicher Erfahrung sich der Objektivität des Denkens nicht mehr ohne weiteres erschließt und dass die Subjekte dieses Denkens theoretisch wie praktisch widersprüchlichen Anforderungen ausgesetzt zu sein scheinen. Diese Anforderungen könnte selbstbewusstes Denken als Zumutungen zurückweisen. Luhmann zieht jedoch die Konsequenz, dass der Subjektbegriff überhaupt überfordert sei und gliedert das Denken in die ihm zugemuteten Bedingungen ein, indem er die Widersprüche, die doch nur dem Denken aufgehen können, als selbsterzeugte Objekte rekonstruiert.

Dieser Entsubjektivierung korrespondiert gesellschaftlich aber gerade das Phänomen fortschreitender Individualisierung: Seit langem ist die Tendenz zur Konzentration des Selbstbewusstseins auf individuelle Befindlichkeiten zu beobachten. Die Philosophie reflektiert dies zum Beispiel in Tugend- und Individualitätsethiken ebenso wie schon in der pragmatischen Analyse von Alltagserfahrungen, die in der Folge der teils alltagssprachlichen teils verwaltungsmäßigen Kritik der philosophischen Terminologie ${ }^{36}$ an die Stelle politischer Theorie gesetzt wird.

${ }^{33}$ G.W.F. Hegel, Wissenschaft der Logik. Lehre vom Begriff, a.a.O., S. $175 \mathrm{f}$.

${ }^{34}$ Als „Selbst` der Selbstreferenz“ fungieren dann „Elemente, [...] Prozesse, [...] das System selbst“. Vgl. Niklas Luhmannn, Soziale Systeme, a.a.O., S. 606.

${ }^{35}$ Peter V. Zima, Theorie des Subjekts, a.a.O., S. 325.

${ }^{36}$ Dies kritisiert auch Henrich, „Fichtes ursprüngliche Einsicht“, a.a.O., S. 229. 
Auch Luhmann registriert die Tendenz, das Individuum zum „Letztentscheider in allen Angelegenheiten“, nach Maßgabe individueller „Meinung, [...] Interesse, [...] Anspruch, [...] Lust ${ }^{\text {“ }}{ }^{37} \mathrm{zu}$ machen. Dem entspreche es, dass die Individuen zu keinem sozialen System dazugehörten und sich so über individualisierte Ansprüche entschädigten. ${ }^{38}$ Die Individualisierung führe aber umgekehrt auf die Frage, „wie psychische Systeme [...] von Moment zu Moment, den ,Strom' ihres ,Bewußtseinslebens', so einrichten können, daß ihre Geschlossenheit mit einer Umwelt sozialer Systeme kompatibel ist“"39. Freiheit wird auf bloße Kontingenz reduziert ${ }^{40}$ und Subjektivität auf den überlebenswichtigen Impuls, das Selbstbewusstsein in die Umgebungsbedingungen einzupassen. Individualität ist die funktionalisierte Gestalt ${ }^{41}$ von Subjektivität und schlägt daher gegen diese um: Die Subjektivität, die keinen allgemeinen Anspruch an die Sachen macht, sondern sich individuell und von Moment zu Moment in sie versenkt, gibt nicht ihnen seine Form, sondern fügt sich selbst in deren Gestalt ein. Faktisch verschwimmt hier Subjektivität in einem stets beweglichen wechselseitigen Bestimmungsverhältnis von Individuum und äußeren Bedingungen. Insofern hätte Luhmann recht gehabt.

Nach Luhmanns Bild sind die Menschen für die Gesellschaft Umwelt, ${ }^{42}$ je eigene Systeme, die gegeneinander und gegen die Gesellschaft ,opak ${ }^{63}$ blieben. Der Differenzierungsprozess der Gesellschaft verlaufe in der Umwelt der Menschen. Deshalb könnten diese ihn nicht kontrollieren, seien aber derart funktional angeschlossen, dass sie als ,psychisch internalisierte soziale Artefakte ${ }^{\text {‘4 }}$ gelten könnten und sich schließlich je selbst zur Umwelt werden, weil ihre soziale und ihre psychische Systemreferenz nicht mehr zur Deckung zu bringen seien. Aus dem Bild, dem, sobald es überhaupt etwas trifft, ein Zustand

${ }^{37}$ Niklas Luhmann, Soziale Systeme, a.a.O., S. S. 633.

${ }^{38}$ Vgl. Niklas Luhmann, Soziale Systeme, a.a.O., S. 365.

${ }^{39}$ Niklas Luhmann, Soziale Systeme, a.a.O., S. 348.

${ }^{40}$ Vgl. Niklas Luhmann, „Soziologie der Moral“, a.a.O., S. 99f. und S. 118.

${ }^{41} \mathrm{Vgl}$. Niklas Luhmann, Soziale Systeme, a.a.O., S. 350.

${ }^{42}$ Vgl. Niklas Luhmann, „Soziologie der Moral“, a.a.O., S. 82f., S. 97f.; Niklas Luhmann, Soziale Systeme, a.a.O., 191, S. $551 \mathrm{f}$.

${ }^{43}$ Vgl. Niklas Luhmann, „Soziologie der Moral“, a.a.O., S. 98. Aufgabe von Gesellschaftstheorie wäre es nicht, den Verhältnissen einen opaken Schimmer zu verleihen; den haben sie ohnehin, seit die bestimmenden Formelemente von Gesellschaft unpersönlicher Art sind und aus der unmittelbaren Erfahrung erst rekonstruiert werden müssen. So reproduziert Luhmann in theoretischem Jargon das, was der unmittelbaren, der Erkenntnis fernsten, Erfahrung gegeben ist. - Dass derart opake Zustände zu Zweifeln an der Subjektivität Anlass geben können, hat Karen Gloy, „Der Begriff des Selbstbewußtseins bei Kant und Fichte“, a.a.O., S. 125, festgehalten: „Die [...] Hypostasierung und Verabsolutierung des Ich zum schlechthin unbedingten, absoluten Wesen mußte zwangsläufig zur Zielscheibe der Kritik werden angesichts der gegenwärtigen Erfahrung ökologischer, ökonomischer und politischer Krisen, die nicht nur die historische Bedingtheit und Abhängigkeit des Ich erkennen lassen, sondern auch seine Ausgeliefertheit an unkontrollierbar gewordene und nicht selten anonymisierte Prozesse.“ - Das gedoppelt Apodiktische „mußte zwangsläufig" wäre freilich zum problematischen „konnte“ zu ermäßigen, denn auch die ,Unkontrollierbarkeit" ist ein Schein, und was ,ausgeliefert 'ist, muss es geben. Wenngleich Subjektivität als absolute grundsätzlich erschüttert wurde, konnte doch kritisch an Subjektivität als Prinzip festgehalten werden; dies jedoch nicht ohne eine grundsätzliche gesellschaftstheoretische Erklärung jener, ökologischen, ökonomischen und politischen Krisen`. Unabhängig von solcher Erklärung bleibt Subjektivität ein Euphemismus für Selbsttäuschung.

${ }^{44}$ Vgl. Niklas Luhmann, Soziale Systeme, a.a.O., S. 551. 
zerrütteten Selbstbewusstseins in Wirklichkeit entspricht, ließe sich eine kritische Theorie der Gesellschaft entwickeln. Diese wäre die theoretische Form intellektueller Selbstbehauptung, die ihrerseits Bedingung - wenngleich nicht Grund - auch des praktischen Festhaltens an Selbstbestimmung ist. Die gedankliche Anstrengung, Erfahrungen durch Vernunft zu beurteilen und so an der Identität des Selbstbewusstseins wenigstens kontrafaktisch festzuhalten, sich nicht irr machen zu lassen, bezeichnet Luhmann jedoch rundweg als ,primitiv ${ }^{65}$, weil sie die Realität nicht im Sinne von deren Funktionalität zu rekonstruieren vermöchte. ${ }^{46}$ Die geforderte Rekonstruktion, in der das Individuum Luhmann zufolge seine Identität nur durch strategisches ,Copieren ${ }^{‘ 47}$ von Anderen bewahrt, wäre aber nicht der Zustand elaborierter Wissenschaft, sondern der der sinnlichen Gewissheit, wie sie erscheint, nachdem der Geist in sie zurückkehrt ist: In ihr ist nichts mehr wahr, aber dafür alles wirklich. „Daraus erheben kann er [der Geist; M.St.] sich nicht mehr, da er alle Widersprüche in sich zurückgenommen hat, bzw. sie dort, wo sie sich scheinbar doch zeigen, glücklich als Vermittlung erlebt. Das Bewußtsein weiß nichts mehr, hat aber alles, was es vergaß, als ein Ensemble von Zitaten und Paraphrasen in sich aufgehoben. Diese im wiedererreichten Anfang vollendete Schöpfungsgeschichte des menschlichen Geistes, wie er jetzt erscheint, kulminiert im definitiven Satz: ,Im Anfang ist die Kopie.' So sehe ich das. “ 48

\footnotetext{
${ }^{45}$ Vgl. Niklas Luhmann, „Soziologie der Moral“, a.a.O., S. 122.

${ }^{46}$ Funktionalität wird zum einzigen Maßstab der Theorie und ihres Gegenstandes. Vgl. Niklas Luhmann, „Soziologie der Moral“, a.a.O., S. 92. Rationalität ist bei Luhmann stets relative Systemrationalität, also fast ein Synonym für Funktionalität. Vgl. Niklas Luhmann, Soziale Systeme, a.a.O., S. 645. - Vernünftige Reflexion könne schon deshalb nicht Maßstab sein, weil sie in der Gesellschaft wirkungslos sei, die allein durch Kommunikation über Kommunikation Reflexivität erzeuge; wohl könnten solche reflexiven Prozesse auch gesellschaftliche Strukturen ändern, so dass keineswegs der Fortbestand des Gleichen vorherzusagen sei. Allerdings sind die Zwecke, durch die Veränderungen bestimmt werden, nach Luhmann nie notwendig vernünftig, sondern immer durch Kommunikation erzeugt. Vgl. Niklas Luhmann, Soziale Systeme, a.a.O., S. 610-612.

${ }^{47}$ Vgl. Niklas Luhmann, Soziale Systeme, a.a.O., S. 366.

48 Robert Menasse, Phänomenologie der Entgeisterung. Geschichte des verschwindenden Wissens, Frankfurt am Main 1995, S. $86 f$.
} 